\title{
Allergy in Children with Functional Constipation and Irritable Bowel Syndrome
}

\author{
Carlo Caffarelli, ${ }^{1,}$ Dora Di Mauro, ${ }^{1}$ Marilena Garrubba, ${ }^{1}$ and Carla Mastrorilli ${ }^{1}$ \\ ${ }^{1}$ Clinica Pediatrica, Dipartimento di Medicina Clinica e Sperimentale, University of Parma, Parma, Italy \\ "Corresponding author: Carlo Caffarelli, Clinica Pediatrica, Dipartimento di Medicina Clinica e Sperimentale, University of Parma, Parma, Italy. Tel: +39-0521702207, Fax: \\ +39-0521702830, E-mail: carlo.caffarelli@unipr.it
}

Received 2015 December 30; Accepted 2016 June 21.

\begin{abstract}
Context: Functional constipation (FC) and irritable bowel syndrome (IBS) represent very common pediatric functional gastrointestinal disorders (FGIDs). Controversial results have suggested a potential role of food allergy as a trigger of functional bowel symptoms.

Evidence Acquisition: This review summarizes the literature regarding the role of allergic diseases in children with FC and IBS and discusses the hypothesis of the pathogenesis of constipation due to cow's milk protein allergy (CMPA). We searched systematic reviews, guidelines, or original data in PubMed, MEDLINE, and the Cochrane central register of controlled trials.

Results: The pathogenesis of FGIDs remains elusive and is likely multifactorial. Among these factors, adverse reactions to food may play a pathogenic role. Some features, such as abnormal bowel motility, visceral hypersensitivity, and changes in mucus composition caused by inflammation of the gastrointestinal wall, have been found both in IBS or FC and in food allergy. Since 1978, an increasing number of reports have suggested a relationship between CMPA and FC. Two randomized controlled studies conducted in children showed that CMPA may induce chronic FC; one study indicated that fermentable oligosaccharide, disaccharide, and monosaccharide polyols (FODMAP) foods may play a role in triggering IBS.

Conclusions: Food allergy in children with chronic constipation should be identified using an oral food challenge after being on a diet free of cow's milk. A diet low in FODMAPs might also be recommended for children with IBS. This approach could be suggested for children with chronic FC and IBS, especially when they do not respond to standard treatment. However, it should also be considered that a minority of patients with FC or IBS could respond to an elimination diet. Further studies are needed to understand the complex pathogenic mechanisms of FGIDs; they also might be helpful to recognize markers for identifying children with IBS and FC caused by foods and to improve their management.
\end{abstract}

Keywords: Constipation, Cow's Milk Allergy, Food Allergy, Functional Gastrointestinal Disorders, Irritable Bowel Syndrome, Children

\section{Context}

Functional constipation (FC) and irritable bowel syndrome (IBS) represent very common functional gastrointestinal disorders (FGIDs) in children and have a worldwide pooled prevalence of $3 \%-16 \%$ and $13.5 \%$, respectively $(1,2)$. The Rome III Criteria were used to define FC and IBS (Box 1) $(3,4)$.

The origin of IBS and FC remains uncertain. They are likely influenced by both a genetic predisposition and environmental agents. Their occurrence has been associated with several factors, such as infection, food allergy/intolerance, immune system dysfunction, visceral hypersensitivity, dysregulation of the central and enteric nervous systems, and abnormal gut motility. Previous studies have shown controversial results regarding the potential role of food allergy/intolerance as a trigger of FC and IBS (2). This review summarizes clinically important findings from the viewpoint of a busy clinician who deals with the role of food reactions in IBS or FC in pediatric patients.

\section{Evidence Acquisition}

This review was obtained in part by searching studies published from 1978-November 2015 in PubMed, MEDLINE, and the Cochrane central register of controlled Trials. We used the following keywords: "children," "functional constipation," "cow's milk allergy," "food allergy," “allergy," and "irritable bowel syndrome". The search was restricted to papers published in English. We considered systematic reviews, guidelines, or original data. References from selected papers were also examined to find additional relevant articles. We retrieved 1568 articles. The titles and abstracts of these articles were reviewed independently by two of the authors. We have not analyzed all the papers in detail. To increase the clinical relevance of this review, we chose to cite randomized controlled studies conducted in 
Box 1. Rome III Diagnostic Criteria for irritable Bowel Syndrome and Functional Constipation

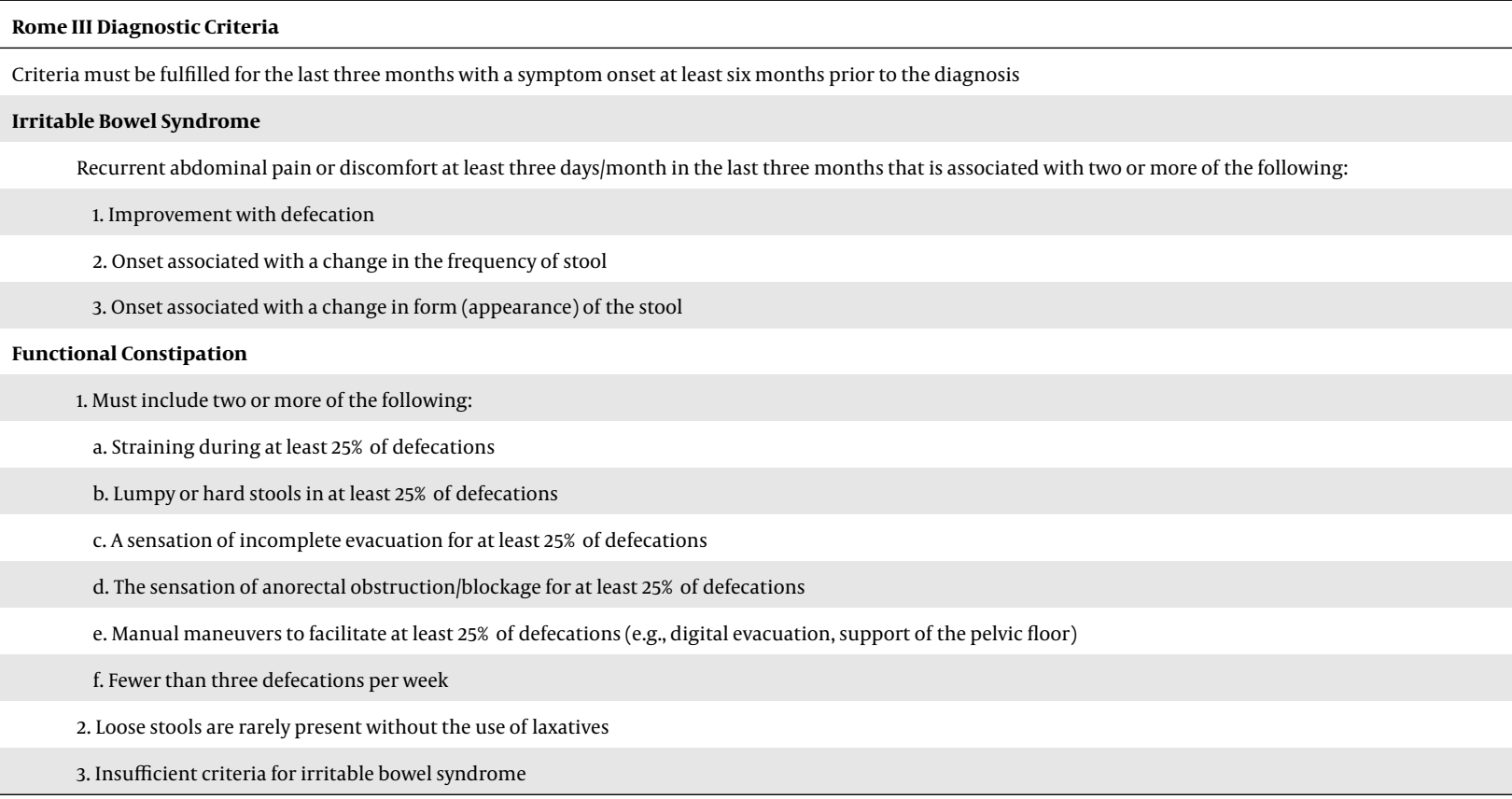

pediatric patients and also other articles on clinically important issues.

\section{Results}

\subsection{Common Mechanisms}

The pathogenesis of FGIDs remains elusive and is likely multifactorial. Among these factors, adverse reactions to food may play a pathogenic role. Some features, such as abnormal bowel motility (slow, fast, or uncoordinated), visceral hypersensitivity, and changes in the mucus composition caused by inflammation of the gastrointestinal wall, have been found both in IBS or FC and in patients with food allergy $(5,6)$. It has also been reported that both cow's milk protein allergy (CMPA) and FGIDs could be associated with increased intestinal permeability and inflammation $(7,8)$. A Th2 immune response has been observed in atopic diseases and FGIDs (9). Some studies have demonstrated that there is overlap between immune system inflammation in FGIDs and in atopic conditions (10). Overall, these data support the hypothesis that FC, IBS, and their related low-grade inflammation might be the expression of a systemic allergic (or "atopic") disorder induced by food $(11,12)$. Accordingly, the allergic inflammation of the rectal wall induced by CMPA results in a higher pressure of the anal sphincter as evidenced by anorectal manometry, which may be one cause of constipation (13-16).
Food allergy due to either IgE- or non-IgE-mediated mechanisms is commonly thought to elicit gut mucosa inflammation. The infiltrate is characterized by mast cells (MCs), eosinophils, and $\mathrm{T}$ and $\mathrm{B}$ lymphocytes that are present at different sites along the gut (17). MCs are regarded as key effector cells of both immediate and delayedtype hypersensitivity reactions (18), as MCs are in close proximity to enteric neurons and release a variety of mediators, which influence nerve function, leading to abnormal gut motility $(19,20)$. Borrelli et al. (13) clearly showed that children with chronic constipation related to food allergy exhibit an increase in the density of MCs in the rectal mucosa and near the submucosal rectal nerve endings, which are correlated with abnormalities in anorectal motility. Biopsies have revealed that allergic manifestations and inflammation of the rectal mucosa more frequently coexist in children with FC who responded to a cow's milk-free diet in comparison with children who did not respond $(14,21)$. Children with a CMPA who also suffered from chronic, refractory constipation were described along with allergic rectitis or colonic lymphoid nodular hyperplasia in various parts of the gastrointestinal mucosa and increased density of intraepithelial $\gamma \delta+$ T cells (14). Turunen et al. (15) found lymphoid nodular hyperplasia as the most noticeable endoscopic finding in half (46\%) of the children with FC, occurring in patches in the colon. Children with a positive cow's milk challenge showed a sig- 
nificantly higher density of intraepithelial $\gamma \delta+$ T cells $(\mathrm{P}<$ 0.001 ) in the biopsy samples of the terminal ileum as compared with the control participants.

\subsection{The Association of Atopic Diseases With IBS or FC}

Several studies in adults have shown a link between functional bowel disorders and asthma, but contrasting results have been reported in children on the relationship between atopic diseases and IBS or FC.

Previous investigations reported increased airway responsiveness to inhaled methacholine and/or reversibility with bronchodilators in IBS patients with no clinical evidence of asthma or other atopic diseases compared to controls $(22,23)$. IBS has also been reported to be more common in asthmatic adults than in the general population; the risk of IBS was reduced with oral steroids in asthma patients (24). Recently, Leander et al. (25) showed that constipation was associated with asthma and wheezing symptoms in preschool children. In a longitudinal birth cohort study, Kiefte-de Jong et al. (26) found that wheezing symptoms and FC frequently coexist in preschool children. No significant association was identified between the symptoms of atopic dermatitis and FC. However, the association between wheezing and constipation or IBS was not independent but instead was largely explained by the child's exposure to infections and the associated antibiotic use.

In children with chronic constipation caused by food intolerance, a history of food intolerance, bronchospasm and atopic eczema was more common than in patients with constipation unrelated to food intolerance (16).

In contrast, IBS is not significantly more prevalent in children with asthma in comparison with those who are free of allergic symptoms (27), which could indicate that asthma may precede the onset of IBS. If this is the case, the presence of MC colonic infiltrate in patients with IBS may support the view that IBS may be a consequence of a chronic systemic inflammation. Moreover, no difference in the frequency of constipation between allergic children and controls was identified, but other gastrointestinal symptoms, such as diarrhea and vomiting, were more prevalent in children with atopic eczema than in healthy children (27). Simeone et al. (28) also showed no association between constipation and atopy in young children, whereas a small increase in the prevalence of IBS over time was observed in participants with asthma who were 5 years of age.

\subsection{Food Allergy and FC}

CMPA was first suggested as a cause of constipation in 1978 (29). In an open study published in 1995, Iacono et al. (21) found 21 (78\%) out of 27 children affected by constipation and CMPA improved after a CMP elimination diet. They also reported that 15 (71.4\%) constipated patients with CMPA (proven by a withdrawal-challenge test) had one or more positive laboratory tests (specific IgG to beta-lactoglobulin, total IgE antibodies, and circulating eosinophils). They hypothesized that a cow's milk intolerance may cause severe perianal lesions with pain on defecation and consequent constipation.

These early findings have been confirmed by randomized controlled studies. Iacono et al. (30) conducted a double-blind crossover randomized, controlled trial in 65 children from 11 - 72 months of age with chronic constipation who did not improve with lactulose. All 44 (68\%) children who experienced symptom remission by substituting cow's milk for soy milk for two weeks participated in a double-blind, placebo-controlled challenge with cow's milk. In all of these patients, constipation reappeared after 5 - 10 days of this diet. The symptoms disappeared in all patients after returning to the cow's milk-free diet. Responders were significantly more likely to have anal fissures and erythema or edema, positive serum CM-specific IgE, inflammation of the rectal mucosa, and a higher frequency of coexistent allergic rhinitis, dermatitis, or bronchospasm. Finally, all the children who were enrolled in this trial had been referred to the study center because they were likely to have a food allergy. Therefore, the results of the study may overestimate the frequency of CMPA in resistant FC.

Recently, Dehghani et al. (31) performed an open, randomized controlled study comparing two groups that each consisted of 70 children who followed a four-week cow's milk-free diet or a diet that included cow's milk. All children suffered from chronic functional constipation that was unresponsive to a corrective laxative treatment. Fifty-six (80\%) children who followed the restricted diet and $47 \%$ of the control children improved $(\mathrm{P}<0.0001)$. A two-week cow's milk challenge was positive in 24 (34\%) patients who were cured on the elimination diet. Only one patient had a positive skin prick test to cow's milk. Moreover, case series of children with FC associated with CMPA have been reported. Daher et al. (14) found that in 7 (28\%) of 25 children, constipation was relieved after a four-week, milkfree diet, and the participants relapsed within two to three days following a challenge with cow's milk. This response was not associated with an IgE-mediated mechanism, since there was no difference between children with CMPA and children without allergy in serum IgE levels to cow's milk extract and proteins. Iacono et al. (16) studied 36 children with chronic constipation between the ages of 9 months to 10 years who went on four-week cow's milk-free diet or a restricted diet. Seventeen children improved on this diet; 14 avoided only cow's milk, while 3 restricted multiple foods 
and were randomized to receive a two-week double-blind placebo controlled challenge with cow's milk or ass's milk as placebo. In all children, the cow's milk challenge produced constipation within five days. Cow's milk or multiple foods in three patients were again excluded from the diet, and all patients experienced symptom improvement.

Turunen et al. (15) showed that remission of constipation occurred in 29 ( $83 \%$ ) of 35 children aged $3-15$ years who followed a four-week cow's milk elimination diet. Constipation and/or other gastrointestinal or skin symptoms occurred in 12 (34\%) children during a four-week cow's milk challenge.

El-Hodhod et al. (32) studied 27 children with chronic FC who did not respond to two months of laxative therapy. Twenty-one (77\%) improved after the elimination of cow's milk and dairy products for a one-month period; constipation recurred in these patients during a two-week open challenge with cow's milk. Serum-specific IgE antibodies to whole cow's milk and beta-lactoglobulin were also significantly higher in constipated patients compared with healthy controls. Tolerance to cow's milk was achieved after 12 months of a cow's milk free-diet in $88 \%$ of these cases.

Irastorza et al. (33) found in an open-label crossover study that 27 (39\%) patients responded to a three-week CMP-elimination diet. Afterwards, all of them experienced constipation within two to five days during the three-week cow's milk challenge. No significant difference was noted between the group of responders and non-responders in regards to their atopic/allergic history or laboratory results.

Other case series have investigated the association of FC not only with cow's milk allergy but also with allergy to other foods. Borrelli et al. (13) reported that 18 (54\%) of 33 children, aged 1 - 10 years, with refractory chronic constipation to laxatives, responded to a six- to -eight-week elimination diet for cow's milk, egg, and soy proteins. Doubleblind, placebo-controlled challenges with cow's milk, egg, and soy followed by a two-week open challenge were carried out in the children who responded to the diet. All 18 children had a positive food challenge within 2 - 14 days. Cow's milk induced constipation in 10 children; soy in 2; egg in 4; and egg, soy, and cow's milk in 2.

Syrigou et al. (34) showed that constipation improved in 28 of 32 children after an eight-week diet free of foods (wheat, egg, cow's milk, rice, corn, soy) whose specific IgE levels, skin prick tests, or atopy patch tests were positive. Twenty-seven responders had positive patch tests, and eight also had positive specific IgE and/or skin prick tests. One responder had a negative patch test to CMP but a positive specific IgE and skin prick test to CMP. The high diagnostic accuracy of the patch test has not been shown in other reports (35). A relapse of constipation was noted af- ter an open oral food challenge in all 28 children. Tolerance to food allergens was achieved in 25 of 28 cases after 12 months of avoidance.

Although the number of reports from different investigations confirming the association of constipation with CMA is increasing, not all studies are in agreement. In an unselected pediatric population, Simeone et al. (28) showed that any of 11 children with constipation refractory to osmotic laxatives did not respond to a four-week cow's milk elimination diet. Moreover, the prevalence of atopy in constipated children was not different from that in children without constipation.

Loening-Bauche et al. (36) reported that only $2 \%$ of children younger than two years of age with functional constipation and a previous history of CMPA who went on a 14-day CMP-free diet experienced symptom resolution. Bergmann et al. (37) strongly disagreed with the common practice of restricting $\mathrm{CM}$ in young infants with common, short-lived gastro-intestinal symptoms (colic, constipation, and gastroesophageal reflux). Their prospective study screened infants younger than six months of age who presented with specific gastrointestinal pathologies. No infants with persistent gastrointestinal symptoms could be identified; therefore, these patients might not need allergy testing or a diagnostic CM-free diet.

In 2010, the opinion of the NICE guideline (38) was that there was some evidence for excluding cow's milk from the diet to improve constipation. However, the quality of studies was poor, and the selection of participants was biased. The European society for Pediatric Gastroenterology, Hepatology, and Nutrition/North American society for pediatric gastroenterology, hepatology, and nutrition guidelines (39) concluded that evidence of an association between FC and CMPA in children was conflicting. There was no rationale for allergy testing in children with constipation, and a two- to four-week restricted diet as a firstline therapeutic approach for young children with constipation was not recommended unless in children with intractable constipation. In 2014, a review by Miceli Sopo et al. (40) evaluated 10 studies that enrolled 505 total patients with chronic constipation and concluded that the benefit of a cow's milk-free diet varied from $28 \%$ - 78\% depending on the study population. An oral food challenge was performed in all studies, and two of them were randomized. The authors concluded that there was evidence that supported a two- to four-week cow's milk elimination diet in children with functional constipation not only when standard treatments have failed but also as a first-line therapeutic strategy. 


\subsection{IBS and Food Intolerance}

The notion of IBS in childhood is quite recent (41) and therapeutic strategies based on avoidance of the offending foods have been deduced from evidence of the efficacy of this procedure in adult studies. The pathogenic role of foods in childhood has been confirmed by studies reporting that children with IBS identify specific foods that are responsible for their symptoms and impair their quality of life. Carlson et al. (42) studied suspected food allergy in 25 child-parent pairs using a questionnaire and focus groups. Most children had IBS (40\%) or abdominal migraine (36\%). The median number of offending foods was 11 . Spicy foods, pizza, and cow's milk were the most frequently identified foods that induced gastrointestinal symptoms. Coping strategies for reducing symptoms included eating smaller portions, modifying the food, or avoiding the food altogether.

Conventional allergy tests often fail to identify food triggers, which suggests that the adverse reaction to food is non-immunologically mediated (food intolerance) (43, 44). The diagnosis of food intolerance largely relies on the resolution of symptoms during a restricted diet and then the onset of a reaction following a food challenge. Several types of elimination diets, such as few-food diets or diets free of chemicals, have been suggested for treating patients with IBS. However, evidence that these diets improve IBS in children is currently lacking.

Increasing attention has been paid to food as a pathogenic trigger in IBS after recent randomized controlled studies $(45,46)$ have supported the existence of a subgroup of adults with IBS who benefit from diets with low fermentable oligosaccharide, disaccharides, and monosaccharide polyols (FODMAPs). FODMAPs are shortchain carbohydrates that are fermentable by colonic bacteria and are poorly absorbed. They include foods containing fructose (honey, apple, apricot, and avocado), lactose (cow's milk), fructooligosaccharides (wheat), galactooligosaccharides (cabbage, legumes, garlic, and leek), and polyols (pear, apricot, corn, cauliflower, and mushrooms). FODMAPs may increase the osmotic pressure in the large intestine and favor bacterial fermentation and gas production, causing increased luminal distention and abnormal motility and resulting in symptoms such as abdominal bloating, pain, and discomfort. Gomara et al. (47) performed a fructose breath test in 32 children with FGIDs. All patients experienced an exacerbation of their intestinal symptoms during the three-hour fructose breath test irrespective of their breath test results. The breath tests were positive in 11 patients. These participants went on a restricted fructose diet; 9 (81\%) reported improvement in their symptoms after two weeks and also after two months. Escobar et al. (48) performed a fructose breath test in 222 children with functional abdominal pain. They found positive results in 121 (54\%) instances. All children with a positive breath test went on a low-fructose diet, and 93 (77\%) of them reported a complete resolution of their symptoms. The causative role of FODMAPs in childhood IBS was also shown by a double-blind crossover trial conducted by Chumpitazi et al. (49). In their 54 participants, two days on a low FODMAP diet containing $0.15 \mathrm{~g} / \mathrm{kg} /$ day (with a maximum of $9 \mathrm{~g} /$ day) (50) significantly decreased the frequency of abdominal pain and also reduced breath hydrogen production compared with a typical American childhood diet. Furthermore, at baseline, responders to the diet had a microbiome composition along with greater saccharolytic capacity than those who did not respond.

The importance of food components as possible triggers of IBS has been particularly stressed for wheat. Mullin et al. (51) recently reviewed the dietary management of IBS patients and pointed out how wheat may act as symptom inducer for several reasons: high fructans content and other members of the family of highly fermentable FODMAP; autoimmune (e.g., celiac) disorder trigger; or IgE and non IgE-mediated allergenicity. In this context, nonceliac gluten sensitivity is gaining attention (52). In addition, there is no evidence that a lactose-free diet may benefit children with IBS $(53,54)$.

\section{Conclusions}

Most patients with FGIDs are certain that particular foods produce their symptoms and would like to know what foods they should avoid. We found that two randomized controlled studies that were conducted in children suggested that CMPA may induce chronic FC; another study indicated that FODMAP foods may have a role in triggering IBS. It is unclear whether IgE tests for CMP are helpful to identify children with FC due to cow's milk. No other laboratory tests have been shown to be useful for diagnosing food allergy/intolerance in children with FC or IBS. Therefore, it should be considered that the role of food in chronic constipation among pediatric patients should be established by a two- to four-week cow's milk-free diet followed by an oral food challenge (55). Cow's milk tolerance is often achieved after 12 months of a cow's milkfree diet. Therefore, children should periodically perform a food challenge to ascertain whether a tolerance has been reached. Similarly, a diet low in FODMAPs might be proposed in children with IBS. Only a minority of patients with FC or IBS respond to elimination diets. However, considering the short duration of a restricted diet (whose impact on nutrition should be managed by a dietician)(56) and its easy adherence, this dietetic approach might be proposed 
for children with chronic FC and IBS, especially when standard treatment has failed (57).

Further studies will be needed to understand the complex pathogenic mechanisms of FGIDs. They could also be helpful to recognize markers for identifying children with IBS and FC caused by foods and to improve their management.

\section{Footnote}

Authors' Contribution: Study concept and design, analysis and interpretation of data, and critical revision: Carlo Caffarelli, Dora Di Mauro, Marilena Garrubba, and Carla Mastrorilli.

\section{References}

1. Loening-Baucke V. Prevalence, symptoms and outcome of constipation in infants and toddlers. J Pediatr. 2005;146(3):359-63. doi: 10.1016/j.jpeds.2004.10.046. [PubMed: 15756220].

2. Canavan C, West J, Card T. The epidemiology of irritable bowel syndrome. Clin Epidemiol. 2014;6:71-80. doi: 10.2147/CLEP.S40245. [PubMed: 24523597].

3. Rome F. Guidelines-Rome III Diagnostic Criteria for Functional Gastrointestinal Disorders. J Gastrointestin Liver Dis. 2006;15(3):307-12. [PubMed: 17203570].

4. Drossman DA, Dumitrascu DL. Rome III: New standard for functional gastrointestinal disorders.J Gastrointestin Liver Dis. 2006;15(3):237-41. [PubMed: 17013448].

5. Shah N, Lindley K, Milla P. Cow's milk and chronic constipation in children. N Engl J Med. 1999;340(11):891-2. [PubMed: 10084907].

6. Scaillon M, Cadranel S. Food allergy and constipation in childhood: how functional is it?. Eur J Gastroenterol Hepatol. 2006;18(2):125-8. [PubMed: 16394791].

7. Barau E, Dupont C. Modifications of intestinal permeability during food provocation procedures in pediatric irritable bowel syndrome. J Pediatr Gastroenterol Nutr. 1990;11(1):72-7. [PubMed: 2117653].

8. Shulman RJ, Eakin MN, Czyzewski DI, Jarrett M, Ou CN. Increased gastrointestinal permeability and gut inflammation in children with functional abdominal pain and irritable bowel syndrome. J Pediatr. 2008;153(5):646-50. doi: 10.1016/j.jpeds.2008.04.062. [PubMed: 18538790].

9. Walker MM, Powell N, Talley NJ. Atopy and the gastrointestinal tracta review of a common association in unexplained gastrointestinal disease. Expert Rev Gastroenterol Hepatol. 2014;8(3):289-99. doi: 10.1586/17474124.2014.881716. [PubMed: 24450399].

10. Ford AC, Talley NJ. Mucosal inflammation as a potential etiological factor in irritable bowel syndrome: a systematic review. J Gastroenterol. 2011;46(4):421-31. doi: 10.1007/s00535-011-0379-9. [PubMed: 21331765].

11. Jones MP, Walker MM, Ford AC, Talley NJ. The overlap of atopy and functional gastrointestinal disorders among 23,471 patients in primary care. Aliment Pharmacol Ther. 2014;40(4):382-91. doi: 10.1111/apt.12846. [PubMed: 24961872].

12. Collins SM, Piche T, Rampal P. The putative role of inflammation in the irritable bowel syndrome. Gut. 2001;49(6):743-5. [PubMed: 11709500].

13. Borrelli O, Barbara G, Di Nardo G, Cremon C, Lucarelli S, Frediani T, et al. Neuroimmune interaction and anorectal motility in children with food allergy-related chronic constipation. Am J Gastroenterol. 2009;104(2):454-63. doi: 10.1038/ajg.2008.109. [PubMed: 19174791].

14. Daher S, Tahan S, Sole D, Naspitz CK, Da Silva Patricio FR, Neto UF, et al Cow's milk protein intolerance and chronic constipation in children. Pediatr Allergy Immunol. 2001;12(6):339-42. [PubMed: 11846872].

15. Turunen S, Karttunen TJ, Kokkonen J. Lymphoid nodular hyperplasia and cow's milk hypersensitivity in children with chronic constipation. J Pediatr. 2004;145(5):606-11. doi: 10.1016/j.jpeds.2004.06.067. [PubMed: 15520758].

16. Iacono G, Bonventre S, Scalici C, Maresi E, Di Prima L, Soresi M, et al. Food intolerance and chronic constipation: manometry and histology study. Eur J Gastroenterol Hepatol. 2006;18(2):143-50. [PubMed: 16394795].

17. Bischoff S, Crowe SE. Gastrointestinal food allergy: new insights into pathophysiology and clinical perspectives. Gastroenterology. 2005;128(4):1089-113. [PubMed: 15825090].

18. Walker MM, Talley NJ, Prabhakar M, Pennaneac'h CJ, Aro P, Ronkainen $\mathrm{J}$, et al. Duodenal mastocytosis, eosinophilia and intraepithelial lymphocytosis as possible disease markers in the irritable bowel syndrome and functional dyspepsia. Aliment Pharmacol Ther 2009;29(7):765-73. doi: 10.1111/j.1365-2036.2009.03937.x. [PubMed: 19183150].

19. Barbara G, Stanghellini V, De Giorgio R, Corinaldesi R. Functional gastrointestinal disorders and mast cells: implications for therapy. Neurogastroenterol Motil. 2006;18(1):6-17. doi: 10.1111/j.13652982.2005.00685.x. [PubMed: 16371078].

20. Collins SM. The immunomodulation of enteric neuromuscular func tion: implications for motility and inflammatory disorders. Gastroenterology. 1996;111(6):1683-99. [PubMed: 8942751].

21. Iacono G, Carroccio A, Cavataio F, Montalto G, Cantarero MD, Notarbartolo A. Chronic constipation as a symptom of cow milk allergy. $J$ Pediatr. 1995;126(1):34-9. [PubMed: 7815220].

22. White AM, Stevens WH, Upton AR, O'Byrne PM, Collins SM. Airway responsiveness to inhaled methacholine in patients with irritable bowel syndrome. Gastroenterology. 1991;100(1):68-74. [PubMed: 1983850].

23. Yazar A, Atis S, Konca K, Pata C, Akbay E, Calikoglu M, et al. Respiratory symptoms and pulmonary functional changes in patients with irritable bowel syndrome. Am J Gastroenterol. 2001;96(5):1511-6. doi: 10.1111/j.1572-0241.2001.03748.x. [PubMed: 11374691].

24. Huerta C, Garcia Rodriguez LA, Wallander MA, Johansson S. Risk of irritable bowel syndrome among asthma patients. Pharmacoepidemiol Drug Saf. 2002;11(1):31-5. doi: 10.1002/pds.666. [PubMed: 11998549].

25. Leander M, Cronqvist A, Janson C, Uddenfeldt M, Rask-Andersen A Health-related quality of life predicts onset of asthma in a longitudinal population study. Respir Med. 2009;103(2):194-200. doi: 10.1016/j.rmed.2008.09.015. [PubMed: 19046862].

26. Kiefte-de Jong JC, Lebon A, Jaddoe VW, Hofman A, de Jongste JC, Moll HA. Is there an association between wheezing and constipation in preschool children? Explanations from a longitudinal birth cohort. BMJ Open. 2011;1(2):000237. doi: 10.1136/bmjopen-2011-000237. [PubMed: 22021889].

27. Caffarelli C, Coscia A, Baldi F, Borghi A, Capra L, Cazzato S, et al. Characterization of irritable bowel syndrome and constipation in children with allergic diseases. Eur J Pediatr. 2007;166(12):1245-52. doi: 10.1007/s00431-006-0410-y. [PubMed: 17345097].

28. Simeone D, Miele E, Boccia G, Marino A, Troncone R, Staiano A. Prevalence of atopy in children with chronic constipation. Arch Dis Child. 2008;93(12):1044-7. doi: 10.1136/adc.2007.133512. [PubMed: 18562455].

29. Buisseret PD. Common manifestations of cow's milk allergy in children. Lancet. 1978;1(8059):304-5. [PubMed: 75338].

30. Iacono G, Cavataio F, Montalto G, Florena A, Tumminello M, Soresi M, et al. Intolerance of cow's milk and chronic constipation in children. N Engl J Med. 1998;339(16):1100-4. doi: 10.1056/NEJM199810153391602. [PubMed: 9770556]. 
31. Dehghani SM, Ahmadpour B, Haghighat M, Kashef S, Imanieh MH, Soleimani M. The Role of Cow's Milk Allergy in Pediatric Chronic Constipation: A Randomized Clinical Trial. Iran J Pediatr. 2012;22(4):46874. [PubMed: 23429756].

32. El-Hodhod MA, Younis NT, Zaitoun YA, Daoud SD. Cow's milk allergy related pediatric constipation: appropriate time of milk tolerance. Pediatr Allergy Immunol. 2010;21(2 Pt 2):407-12. doi:10.1111/j.13993038.2009.00898.x. [PubMed:19555354].

33. Irastorza I, Ibanez B, Delgado-Sanzonetti L, Maruri N, Vitoria JC. Cow's-milk-free diet as a therapeutic option in childhood chronic constipation. J Pediatr Gastroenterol Nutr. 2010;51(2):171-6. doi: 10.1097/MPG.0b013e3181cd2653. [PubMed: 20453672].

34. Syrigou EI, Pitsios C, Panagiotou I, Chouliaras G, Kitsiou S, Kanariou $\mathrm{M}$, et al. Food allergy-related paediatric constipation: the usefulness of atopy patch test. EurJPediatr. 2011;170(9):1173-8. doi: 10.1007/s00431011-1417-6. [PubMed: 21347849].

35. Caglayan Sozmen S, Povesi Dascola C, Gioia E, Mastrorilli C, Rizzuti L, Caffarelli C. Diagnostic accuracy of patch test in children with food allergy. Pediatr Allergy Immunol. 2015;26(5):416-22. doi: 10.1111/pai.12377. [PubMed: 25808316].

36. Loening-Baucke V. Controversies in the management of chronic constipation.J Pediatr Gastroenterol Nutr. 2001;32 Suppl 1:S38-9. [PubMed: 11321420].

37. Bergmann MM, Caubet JC, McLin V, Belli DC, Schappi MG, Eigenmann PA. Common colic, gastroesophageal reflux and constipation in infants under 6 months of age do not necessitate an allergy work-up. Pediatr Allergy Immunol. 2014;25(4):410-2. doi:10.1111/pai.12199. [PubMed: 24438166].

38. National Collaborating Centre for Women's and Children's Health (UK) . Constipation in children and young people - Diagnosis and management of idiopathic childhood constipation in primary and secondary care. London: RCOG Press; 2010.

39. Tabbers MM, DiLorenzo C, Berger MY, Faure C, Langendam MW, Nurko S, et al. Evaluation and treatment of functional constipation in infants and children: evidence-based recommendations from ESPGHAN and NASPGHAN. J Pediatr Gastroenterol Nutr. 2014;58(2):25874. [PubMed: 24345831].

40. Miceli Sopo S, Arena R, Greco M, Bergamini M, Monaco S. Constipation and cow's milk allergy: a review of the literature. Int Arch Allergy Immunol. 2014;164(1):40-5. doi: 10.1159/000362365. [PubMed: 24853450].

41. El-Matary W, Spray C, Sandhu B. Irritable bowel syndrome: the commonest cause of recurrent abdominal pain in children. Eur J Pediatr. 2004;163(10):584-8. doi: 10.1007/s00431-004-1503-0. [PubMed: 15290263].

42. Carlson MJ, Moore CE, Tsai CM, Shulman RJ, Chumpitazi BP. Child and parent perceived food-induced gastrointestinal symptoms and quality of life in children with functional gastrointestinal disorders. JAcad Nutr Diet. 2014;114(3):403-13. doi: 10.1016/j.jand.2013.10.013. [PubMed: 24360501]

43. Monsbakken KW, Vandvik PO, Farup PG. Perceived food intolerance in subjects with irritable bowel syndrome- etiology, prevalence and consequences. Eur J Clin Nutr. 2006;60(5):667-72. doi: 10.1038/sj.ejcn.1602367. [PubMed: 16391571].

44. Wilson K, Hill RJ. The role of food intolerance in functional gastrointestinal disorders in children. Aust Fam Physician. 2014;43(10):686-9.
[PubMed: 25286424].

45. Shepherd SJ, Parker FC, Muir JG, Gibson PR. Dietary triggers of abdominal symptoms in patients with irritable bowel syndrome: randomized placebo-controlled evidence. Clin Gastroenterol Hepatol. 2008;6(7):765-71. doi: 10.1016/j.cgh.2008.02.058. [PubMed: 18456565].

46. Halmos EP, Power VA, Shepherd SJ, Gibson PR, Muir JG. A diet low in FODMAPs reduces symptoms of irritable bowel syndrome. Gastroenterology. 2014;146(1):67-75. doi: 10.1053/j.gastro.2013.09.046. [PubMed: 24076059].

47. Gomara RE, Halata MS, Newman LJ, Bostwick HE, Berezin SH, Cukaj $\mathrm{L}$, et al. Fructose intolerance in children presenting with abdominal pain. J Pediatr Gastroenterol Nutr. 2008;47(3):303-8. [PubMed: 18728526].

48. Escobar MA, Lustig D, Pflugeisen BM, Amoroso PI, Sherif D, Saeed $\mathrm{R}$, et al. Fructose intolerance/malabsorption and recurrent abdominal pain in children. J Pediatr Gastroenterol Nutr. 2014;58(4):498-501. [PubMed: 24667867].

49. Chumpitazi BP, Cope JL, Hollister EB, Tsai CM, McMeans AR, Luna RA, et al. Randomised clinical trial: gut microbiome biomarkers are associated with clinical response to a low FODMAP diet in children with the irritable bowel syndrome. Aliment Pharmacol Ther. 2015;42(4):41827. doi: 10.1111/apt.13286. [PubMed: 26104013].

50. Ong DK, Mitchell SB, Barrett JS, Shepherd SJ, Irving PM, Biesiekierski JR, et al. Manipulation of dietary short chain carbohydrates alters the pattern of gas production and genesis of symptoms in irritable bowel syndrome. J Gastroenterol Hepatol. 2010;25(8):1366-73. doi: 10.1111/j.1440-1746.2010.06370.x. [PubMed: 20659225].

51. Mullin GE, Shepherd SJ, Chander Roland B, Ireton-Jones C, Matarese LE. Irritable bowel syndrome: contemporary nutrition management strategies. JPEN J Parenter Enteral Nutr. 2014;38(7):781-99. doi: 10.1177/0148607114545329. [PubMed: 25085503].

52. Sapone A, Bai JC, Ciacci C, Dolinsek J, Green PH, Hadjivassiliou M, et al. Spectrum of gluten-related disorders: consensus on new nomenclature and classification. BMC Med. 2012;10:13. doi: 10.1186/1741-7015-1013. [PubMed: 22313950].

53. Dearlove J, Dearlove B, Pearl K, Primavesi R. Dietary lactose and the child with abdominal pain. BrMed J (Clin Res Ed). 1983;286(6382):1936. [PubMed: 6407644].

54. Lebenthal E, Rossi TM, Nord KS, Branski D. Recurrent abdominal pain and lactose absorption in children. Pediatrics. 1981;67(6):828-32. [PubMed: 7195004]

55. Caffarelli C, Rico S, Rinaldi L, Povesi Dascola C, Terzi C, Bernasconi S. Blood pressure monitoring in children undergoing food challenge: association with anaphylaxis. Ann Allergy Asthma Immunol. 2012;108(4):285-6. doi: 10.1016/j.anai.2012.02.001. [PubMed: 22469454].

56. Giovannini M, D’Auria E, Caffarelli C, Verduci E, Barberi S, Indinnimeo L, et al. Nutritional management and follow up of infants and children with food allergy: Italian Society of Pediatric Nutrition/Italian Society of Pediatric Allergy and Immunology Task Force Position Statement. Ital J Pediatr. 2014;40:1. doi: 10.1186/1824-7288-40-1. [PubMed: 24386882].

57. Nimrouzi M, Sadeghpour O, Imanieh MH, Shams Ardekani M, Salehi A, Minaei MB, et al. Flixweed vs. Polyethylene Glycol in the Treatment of Childhood Functional Constipation: A Randomized Clinical Trial. Iran J Pediatr. 2015;25(2):425. doi:10.5812/ijp.425. [PubMed: 26196006]. 